\title{
Preface for the special issue: modeling, optimization and simulation
}

\author{
Thomas Goetz ${ }^{1} \cdot$ S. Sundar ${ }^{2}$
}

Published online: 7 June 2018

(C) Indian Institute of Technology Madras 2018

Growing importance and usefulness of Industry Academia collaborative research clearly drive the focus on Mathematical Modeling, Optimization and Numerical Simulation today. This Special Issue is the beginning of bringing out a wide spectrum of fine fresh thoughts by some of the esteemed researchers in their respective field of specializations and the challenges therein.

- Optimization and simulation of nonwoven textile production is a challenging problem and the underlying mathematical approach presented here is more rigor and interesting.

- Scaled and fractional Brownian motion through p-variational statistics in order to understand the discriminating measure is a novel idea.

- Special type of fractal interpolation functions with the quality of shape preserving showcase its need in various applications.

- A finite element analysis for the general coupled system of elliptic equations through weighted extended B-Spline put forth the direction of a generic mathematical approach for such class of problems.

- An optimized numerical scheme based on a meshfree particle method (finite pointset method) for a "vision based" pedestrian dynamics simulation is another new direction of thought and shows how the numerical simulation close to the reality.

- In general, the problem of uncertainty quantifications is more complex. Intrusive methods in uncertainty quantifications relating to kinetic theory makes interesting and opens up new mathematical questions.

- Modeling and simulation of ultrasonic beam skewing in polycrystalline materials is another direct industrial problem with a good mathematical flavor.

- Two dimensional and three dimensional Cahn-Hilliard equations numerically solved through the higher order spectral element scheme underline the requirement of such approach.

- Fractional order diffusion and wave equations are hard to solve including setting a sound numerical technique. A spectral Galerkin method for these fractional order equations is a new attempt and brings out how the known well proven methods can be extended to handle such mathematical problems.

Prof. S. Sundar

Department of Mathematics, IIT Madras

Associate Editor, AEAM and Guest Editor for S.I. Modeling, Optimization and Simulation
Thomas Goetz

goetz@uni-koblenz.de

S. Sundar

slnt@iitm.ac.in

1 Mathematik Institut, Universität Koblenz, Koblenz, Germany

2 Department of Mathematics, IIT Madras, Chennai, India 